\title{
Vizing's 2-factor Conjecture Involving Toughness and Maximum Degree Conditions
}

\author{
Jinko Kanno* \\ Department of Mathematics and Statistics \\ Louisiana Tech University \\ Ruston, LA, U.S.A. \\ jkanno@latach.edu
}

\author{
Songling Shan \\ Department of Mathematics \\ Illinois State University \\ Normal, IL, U.S.A. \\ sshan12@ilstu.edu
}

Submitted: Sep 23, 2017; Accepted: Apr 8, 2019; Published: May 3, 2019

(C) The authors. Released under the CC BY license (International 4.0).

\begin{abstract}
Let $G$ be a simple graph, and let $\Delta(G)$ and $\chi^{\prime}(G)$ denote the maximum degree and chromatic index of $G$, respectively. Vizing proved that $\chi^{\prime}(G)=\Delta(G)$ or $\chi^{\prime}(G)=\Delta(G)+1$. We say $G$ is $\Delta$-critical if $\chi^{\prime}(G)=\Delta(G)+1$ and $\chi^{\prime}(H)<\chi^{\prime}(G)$ for every proper subgraph $H$ of $G$. In 1968, Vizing conjectured that if $G$ is a $\Delta$-critical graph, then $G$ has a 2 -factor. Let $G$ be an $n$-vertex $\Delta$-critical graph. It was proved that if $\Delta(G) \geqslant n / 2$, then $G$ has a 2-factor; and that if $\Delta(G) \geqslant 2 n / 3+13$, then $G$ has a hamiltonian cycle, and thus a 2-factor. It is well known that every 2-tough graph with at least three vertices has a 2 -factor. We investigate the existence of a 2 -factor in a $\Delta$-critical graph under "moderate" given toughness and maximum degree conditions. In particular, we show that if $G$ is an $n$-vertex $\Delta$-critical graph with toughness at least $3 / 2$ and with maximum degree at least $n / 3$, then $G$ has a 2 -factor. We also construct a family of graphs that have order $n$, maximum degree $n-1$, toughness at least $3 / 2$, but have no 2 -factor. This implies that the $\Delta$-criticality in the result is needed. In addition, we develop new techniques in proving the existence of 2 -factors in graphs.
\end{abstract}

Mathematics Subject Classifications: 05C38, 05C42

\section{Introduction}

In this paper, we consider only simple, undirected, and finite graphs. Let $G$ be a graph. The notation $\Delta$ is fixed for the maximum degree of $G$ throughout the paper. A $k$-vertex of $G$ is a vertex of degree exactly $k$ in $G$. Denote by $V_{\Delta}$ the set of $\Delta$-vertices in $G$, and

\footnotetext{
*This author is an endowed Upchurch Professor. The Professorship is made available through the State of Louisiana Board of Regents Support Funds.
} 
by $\chi^{\prime}(G)$ the chromatic index of $G$. The graph $G$ is called critical if $\chi^{\prime}(G)>\Delta$ and $\chi^{\prime}(H)<\chi^{\prime}(G)$ for every proper subgraph $H$ of $G$. It is clear that if $G$ is critical then $G$ must be connected. In 1965, Vizing [15] showed that a graph of maximum degree $\Delta$ has chromatic index either $\Delta$ or $\Delta+1$. If $\chi^{\prime}(G)=\Delta$, then $G$ is said to be of class 1 ; otherwise, it is said to be of class 2. Holyer [8] showed that it is NP-complete to determine whether an arbitrary graph is of class 1 . A critical graph $G$ is called $\Delta$-critical if $\chi^{\prime}(G)=\Delta+1$. So $\Delta$-critical graphs are class 2 graphs. Motivated by the classification problem, Vizing studied critical class 2 graphs, or $\Delta$-critical graphs, and made two well-known conjectures.

The first conjecture [16] is on the independence number $\alpha(G)$ of $G$, that is, the size of a maximum independent set in $G$.

Conjecture 1 (Vizing's Independence Number Conjecture). Let $G$ be a $\Delta$-critical graph of order $n$. Then $\alpha(G) \leqslant n / 2$.

Furthermore, Vizing [14] conjectured that the following statement is true.

Conjecture 2 (Vizing's 2-Factor Conjecture). Let $G$ be a $\Delta$-critical graph. Then $G$ contains a 2-factor.

As each cycle $C$ satisfying $\alpha(C) \leqslant|V(C)| / 2$, Conjecture 2 implies Conjecture 1 .

For the Independence Number Conjecture, Brinkmann et al. [2], in 2000, proved that if $G$ is a critical graph, then $\alpha(G)<2 n / 3$; and the upper bound is further improved when the maximum degree is between 3 and 10. Luo and Zhao [11], in 2008, by improving the result of Brinkmann et al., showed that if $G$ is an $n$-vertex $\Delta$-critical graph, then $\alpha(G)<(5 \Delta-6) n /(8 \Delta-6)<5 n / 8$ if $\Delta \geqslant 6$. In 2009, Woodall [17] further improved the upper bound to $3 n / 5$. By restricting the problem to graphs with large maximum degrees, in 2006, Luo and Zhao [10] showed that Vizing's Independence Number Conjecture is true if $\Delta(G) \geqslant n / 2$.

Compared to the progresses on the first Conjecture, the progresses on Vizing's 2-Factor Conjecture has been slow. In 2004, Grünewald and Steffen [7] established Vizing's 2-Factor Conjecture for graphs with the deficiency $\sum_{v \in V(G)}\left(\Delta(G)-d_{G}(v)\right)$ small; in particular, for overfull graphs (graphs of an odd order and with the deficiency $\sum_{v \in V(G)}\left(\Delta(G)-d_{G}(v)\right)<$ $\Delta(G))$. In 2012, Luo and Zhao [12] proved that if $G$ is an $n$-vertex $\Delta$-critical graph with $\Delta \geqslant \frac{6 n}{7}$, then $G$ contains a hamiltonian cycle, and thus a 2 -factor with exactly one component. Continuing the investigation on the existence of a hamiltonian cycle in $\Delta$-critical graphs with "very large" maximum degrees, Luo and Zhao [9] in 2016 showed that an $n$-vertex $\Delta$-critical graph with $\Delta \geqslant \frac{4 n}{5}$ is hamiltonian. The lower bound on $\Delta(G)$ assuring an $n$-vertex $\Delta$-critical graph to be hamiltonian, has been improved to $\frac{2 n}{3}+12$, in [3]. Just finding 2-factors, Chen and Shan [5] proved the following result.

Theorem 1.1 ([5]). Let $G$ be an $n$-vertex $\Delta$-critical graph. Then $G$ has a 2-factor if $\Delta \geqslant n / 2$.

As a measure of graph connectivity and "resilience" under removal of vertices, graph toughness is a useful condition in finding factors in graphs. To be precise, we recall the 
definition of toughness below. The number of components of $G$ is denoted by $c(G)$. Let $t \geqslant 0$ be a real number. The graph is said to be $t$-tough if $|S| \geqslant t \cdot c(G-S)$ for each $S \subseteq V(G)$ with $c(G-S) \geqslant 2$. The toughness $\tau(G)$ is the largest real number $t$ for which $G$ is $t$-tough, or is $\infty$ if $G$ is complete. A toughset in $G$ is a cutset $S \subseteq V(G)$ of $G$ such that $\frac{|S|}{c(G-S)}=\tau(G)$. Enomoto et al. [6] proved the classic result below.

Theorem 1.2 (Enomoto et al. [6]). Every $k$-tough graph has a $k$-factor if $k|V(G)|$ is even and $|V(G)| \geqslant k+1$.

Combining the result in Theorem 1.1 and the result in Theorem 1.2 when restricted to 2-factors, one might wonder - can we get something in between, i.e., is it possible to find 2 -factors in an $n$-vertex $\Delta$-critical graph $G$, under the condition that $\Delta(G)<n / 2$ but $\Delta(G) \geqslant c n$ for some positive constant $c$, and $\tau(G)<2$ but $\tau(G) \geqslant d$ for some positive constant $d$ ? Particularly, we prove the following result.

Theorem 1.3. Let $G$ be an n-vertex $\Delta$-critical graph. Then $G$ has a 2-factor if $\tau(G) \geqslant$ $3 / 2$ and $\Delta \geqslant n / 3$.

The remaining of the paper is organized as follows: in Section 2, we recall some graph terminologies and present several lemmas; in Section 3, we recall Tutte's 2-factor Theorem and develop techniques for showing the existence of 2-factors upon applying Tutte's 2factor Theorem; in Section 4, we prove Theorem 1.3. In the last Section, we construct a family of graphs that have order $n$, maximum degree $n-1$, toughness at least $3 / 2$, but have no 2-factor. This implies that the $\Delta$-criticality in Theorem 1.3 is needed.

\section{Notation and Lemmas}

Let $G$ be a graph. For $x \in V(G)$ we denote by $d_{G}(x)$ the degree of $x$ in $G$. For disjoint subsets of vertices $S$ and $T$ in $G$, we denote by $E_{G}(S, T)$, the set of edges that has one end vertex in $S$ and the other in $T$, and let $e_{G}(S, T)=\left|E_{G}(S, T)\right|$. If $S=\{s\}$ is a singleton, we write $e_{G}(s, T)$ instead of $e_{G}(\{s\}, T)$. If $H \subseteq G$ is a subgraph of $G$, and $T \subseteq V(G)$ with $T \cap V(H)=\emptyset$, we write $E_{G}(H, T)$ and $e_{G}(H, T)$ for notational simplicity. A matching in $G$ is a set of independent edges. If $M$ is a matching of $G$, then let $V(M)$ denote the set of end vertices of the edges in $M$. For $X \subseteq V(G), M$ is said to saturate $X$ if $X \subseteq V(M)$. If $G$ is a bipartite graph with partite sets $A$ and $B$, we denote $G$ by $G[A, B]$ to emphasize the two partite sets.

To prove Theorem 1.3, we present two lemmas below.

Lemma 2.1 (Vizing's Adjacency Lemma). Let $G$ be a $\Delta$-critical graph. Then for any edge $x y \in E(G), x$ is adjacent to at least $\Delta-d_{G}(y)+1 \Delta$-vertices $z$ with $z \neq y$.

The following lemma is a generalization of a result in [10].

Lemma 2.2. Let $G$ be a $\Delta$-critical graph and $T$ be an independent set in $G$. Let $S=$ $V(G)-T$, and let $H=G-E(G[S])$ be the bipartite graph with partite sets $S$ and $T$. 
For each $x \in S$, let $\sigma_{x}$ be the number of non- $\Delta$-degree neighbors of $x$ from $S$. Assume that there are $\delta_{0} \Delta$-vertices in $T$. Then for each edge $x y \in E(H)$ with $x \in S$ and $y \in T$, $d_{H}(y) \geqslant d_{H}(x)+1-\delta_{0}+\sigma_{x}$.

Proof. Let $x y \in E(H)$ with $x \in S$ and $y \in T$. By Vizing's Adjacency Lemma, $x$ is adjacent to at least $\Delta-d_{G}(y)+1 \Delta$-vertices in $G$. As $T$ has $\delta_{0} \Delta$-vertices, we know $x$ is adjacent to at least $\Delta-d_{G}(y)+1-\delta_{0} \Delta$-vertices from $S$. Let $\sigma_{x}$ be the number of all non $\Delta$-degree neighbors of $x$ in $S$. Then, $d_{H}(x)+\Delta-d_{G}(y)+1-\delta_{0}+\sigma_{x} \leqslant d_{G}(x) \leqslant \Delta$. By noting that $d_{G}(y)=d_{H}(y)$, the inequality implies that $d_{H}(y) \geqslant d_{H}(x)+1-\delta_{0}+\sigma_{x}$.

\section{Tutte's 2-factor Theorem and Biased Barriers}

One of the main proof ingredients of Theorem 1.3 is to apply Tutte's 2-factor Theorem under a new setting that we develop in this section.

Let $S$ and $T$ be disjoint subsets of vertices of a graph $G$. Let $D$ be a component of $G-(S \cup T)$. Then $D$ is said to be an odd component (resp. even component) if $e_{G}(D, T) \equiv 1(\bmod 2)\left(\operatorname{resp} . e_{G}(D, T) \equiv 0(\bmod 2)\right)$. Let $\mathcal{H}(S, T)$ be the set of odd components of $G-(S \cup T)$ and let $h(S, T)=|\mathcal{H}(S, T)|$. For $y \in T$, let $\mathcal{H}(y: S, T)=\{D \in$ $\left.\mathcal{H}(S, T), e_{G}(y, D)>0\right\}$ and $h(y: S, T)=|\mathcal{H}(y: S, T)|$. Note that $e_{G}(y, V(G)-(S \cup T)) \geqslant$ $h(y: S, T)$.

Let $\delta(S, T)=2|S|-2|T|+\sum_{y \in T} d_{G-S}(y)-h(S, T)$. It is easy to see that $\delta(S, T) \equiv 0$ (mod 2) for every $S, T \subseteq V(G)$ with $S \cap T=\emptyset$. We use the following criterion for the existence of a 2 -factor, which is a restricted form of Tutte's $f$-factor Theorem.

Lemma 3.1 (Tutte [13]). A graph $G$ has a 2-factor if and only if $\delta(S, T) \geqslant 0$ for every $S, T \subseteq V(G)$ with $S \cap T=\emptyset$.

An ordered pair $(S, T)$ consists of disjoint subsets of vertices $S$ and $T$ in a graph $G$ is called a barrier if $\delta(S, T) \leqslant-2$. By Lemma 3.1, if $G$ does not have a 2-factor, then $G$ has a barrier. We define a special barrier as below.

Definition 1. Let $G$ be a graph without a 2-factor. A barrier $(S, T)$ of $G$ is called a biased barrier if among all the barriers of $G$,

(1) $|S|$ is maximum; and

(2) subject to (1), $|T|$ is minimum.

Properties of a minimum barrier (a barrier such that $|S \cup T|$ is minimum among all the barriers of $G$ ) has been established, for example, in [1,4]. A biased barrier has similar nice properties as given in the lemma below.

Lemma 3.2. Let $G$ be a graph without a 2 -factor, and let $(S, T)$ be a biased barrier of $G$. Then each of the following holds.

(1) The set $T$ is independent in $G$. 
(2) If $D$ is an even component with respect to $(S, T)$, then $e_{G}(T, D)=0$.

(3) If $D$ is an odd component with respect to $(S, T)$, then for any $y \in T, e_{G}(y, D) \leqslant 1$.

(4) If $D$ is an odd component with respect to $(S, T)$, then for any $x \in V(D), e_{G}(x, T) \leqslant 1$.

Proof. Let $U=V(G)-(S \cup T)$ and $z \in T$ be a vertex. By the assumption that $(S, T)$ is a biased barrier, we know that $\delta(S, T-\{z\}) \geqslant 0$. So,

$$
\begin{aligned}
0 & \leqslant \delta(S, T-\{z\})=2|S|-2|T|+2+\sum_{y \in T-\{z\}} d_{G-S}(y)-h(S, T-\{z\}) \\
& =2|S|-2|T|+2+\sum_{y \in T} d_{G-S}(y)-e_{G}(z, T-\{z\})-e_{G}(z, U)-h(S, T-\{z\}) \\
& \leqslant 2|S|-2|T|+2+\sum_{y \in T} d_{G-S}(y)-e_{G}(z, T-\{z\})-e_{G}(z, U)-h(S, T)+h(z: S, T) \\
& =\delta(S, T)+2-e_{G}(z, T-\{z\})-e_{G}(z, U)+h(z: S, T) \\
& \leqslant-e_{G}(z, T-\{z\})-e_{G}(z, U)+h(z: S, T), \quad \text { since } \delta(S, T) \leqslant-2 .
\end{aligned}
$$

This implies that

$$
e_{G}(z, T-\{z\})+e_{G}(z, U)-h(z: S, T) \leqslant 0 .
$$

Because $e_{G}(z, U)-h(z: S, T) \geqslant 0$ always holds, the above inequality particularly implies that

$$
e_{G}(z, T-\{z\})=0 \quad \text { for any } z \in T \quad \text { and } \quad e_{G}(z, U)-h(z: S, T)=0 .
$$

This proves statements (1)-(3).

To show (4), let $D$ be an odd component with respect to $(S, T)$ and let $x \in V(D)$ be any vertex. Then by the assumption that $|S|$ is maximum, we know that $\delta(S \cup\{x\}, T) \geqslant 0$. So,

$$
\begin{aligned}
0 & \leqslant \delta(S \cup\{x\}, T)=2|S|-2|T|+2+\sum_{y \in T} d_{G-(S \cup\{x\})}(y)-h(S \cup\{x\}, T) \\
& =2|S|-2|T|+2+\sum_{y \in T} d_{G-S}(y)-e_{G}(x, T)-h(S \cup\{x\}, T) \\
& \leqslant 2|S|-2|T|+2+\sum_{y \in T} d_{G-S}(y)-e_{G}(x, T)-(h(S, T)-1) \\
& =\delta(S, T)+2-e_{G}(x, T)+1 \\
& \leqslant-e_{G}(x, T)+1, \quad \text { since } \delta(S, T) \leqslant-2 .
\end{aligned}
$$

Hence, $e_{G}(x, T) \leqslant 1$.

Let $G$ be a graph without a 2 -factor and let $(S, T)$ be a biased barrier of $G$. We call $(S, T)$ a good biased barrier of $G$ if $h(S, T)$ is smallest among all biased barriers of $G$.

Lemma 3.3. Let $G$ be a graph without a 2-factor, and let $(S, T)$ be a good biased barrier of $G$. For any $y \in T$, if $h(y: S, T) \geqslant 2$, then for any $D \in \mathcal{H}(y: S, T),|V(D)| \geqslant 3$.

Proof. Let $D \in \mathcal{H}(y: S, T)$ be an odd component of $G-(S \cup T)$. By (4) of Lemma $3.2,|V(D)| \geqslant 3$ if $e_{G}(D, T) \geqslant 3$. So we assume that $e_{G}(D, T)=1$ and assume to 
the contrary that $|V(D)| \leqslant 2$. Let $x$ be the vertex in $D$ if $|V(D)|=1$, and be a vertex in $D$ which is not adjacent to any vertex in $T$ if $|V(D)|=2$. Let $z \in T$ be the vertex such that $e_{G}(D, z)=1$, and let $T^{\prime}=(T-\{z\}) \cup\{x\}$ and $U=V(G)-(S \cup T)$. Let $D_{z}$ be the component of $G-\left(S \cup T^{\prime}\right)$ which contains the vertex $z$. Then since $e_{G}\left(z, D^{\prime}\right)=1$ for any $D^{\prime} \in \mathcal{H}(z: S, T)$ by (3) of Lemma 3.2, we have that

$$
e_{G}\left(D_{z}, T\right)=\left\{\begin{array}{l}
\sum_{D^{\prime} \in \mathcal{H}(z: S, T)-\{D\}}\left(e_{G}\left(D^{\prime}, T\right)-1\right)+e_{G}(x, z), \quad \text { if }|V(D)|=1 \\
\sum_{D^{\prime} \in \mathcal{H}(z: S, T)}\left(e_{G}\left(D^{\prime}, T\right)-1\right)+e_{G}(x, V(D)-\{x\}), \quad \text { if }|V(D)|=2 .
\end{array}\right.
$$

Since $e_{G}\left(D^{\prime}, T\right)$ is odd for any $D^{\prime} \in \mathcal{H}(z: S, T)$, and $e_{G}(x, z)=e_{G}(x, V(D)-\{x\})=1$, we know that $D_{z} \in \mathcal{H}\left(S, T^{\prime}\right)$ is an odd component of $G-\left(S \cup T^{\prime}\right)$. Hence, $h\left(S, T^{\prime}\right)=$ $h(S, T)-h(z: S, T)+1$. So

$$
\begin{aligned}
& \delta\left(S, T^{\prime}\right)=2|S|-2|T|+\sum_{y \in T^{\prime}} d_{G-S}(y)-h\left(S, T^{\prime}\right) \\
= & 2|S|-2|T|+\sum_{y \in T} d_{G-S}(y)+e_{G}(x, V(D-x) \cup\{z\})- \\
& e_{G}(z, U)-h(S, T)+h(z: S, T)-1 \\
= & \delta(S, T)+e_{G}(x, V(D-x) \cup\{z\})-e_{G}(z, U)+h(z: S, T)-1 \\
\leqslant & \delta(S, T) \leqslant-2, \quad \text { since } e_{G}(x, V(D-x) \cup\{z\})=1, \text { and } e_{G}(z, U) \geqslant h(z: S, T) .
\end{aligned}
$$

Thus, $\left(S, T^{\prime}\right)$ is a biased barrier. However, $h\left(S, T^{\prime}\right)=h(S, T)-h(y: S, T)+1 \leqslant h(S, T)-1$, showing a contradiction to the assumption that $(S, T)$ is a good biased barrier.

\section{Proof of Theorem 1.3}

Let $G$ be an $n$-vertex $\Delta$-critical graph such that $\tau(G) \geqslant 3 / 2$ and $\Delta \geqslant n / 3$. We show that $G$ has a 2 -factor.

Since $G$ is $3 / 2$-tough, $\Delta(G) \geqslant \delta(G) \geqslant 3$. Assume to the contrary that $G$ does not have a 2-factor. Then by Tutte's 2-factor Theorem (Lemma 3.1), $G$ has a barrier. Let $(S, T)$ be a good biased barrier of $G$. Since $S$ and $T$ are already fixed, we simply denote $\mathcal{H}(S, T)$ by $\mathcal{H}$. Let $U=V(G)-(S \cup T)$ and let $\mathcal{H}_{k}$ be the set of components $D$ of $G-(S \cup T)$ with $e_{G}(D, T)=k$. Then we have $\mathcal{H}=\bigcup_{k \geqslant 0} \mathcal{H}_{2 k+1}$. For any $y \in T$, let

$$
\begin{aligned}
\mathcal{H}(y) & =\left\{D \in \mathcal{H} \mid e_{G}(y, D)=1\right\}, \\
\mathcal{H}_{1}(y) & =\left\{D \in \mathcal{H}_{1} \mid e_{G}(y, D)=1\right\} .
\end{aligned}
$$

It is clear that $\mathcal{H}_{1}(y) \subseteq \mathcal{H}(y)$. Note also that $\mathcal{H}(y)=\mathcal{H}(y: S, T)$. We use this notation $\mathcal{H}(y)$ for simplicity since $S$ and $T$ are already fixed.

Claim 4.1. $|T| \geqslant|S|+\sum_{k \geqslant 1} k\left|\mathcal{H}_{2 k+1}\right|+2$. In particular, $|T| \geqslant|S|+2$. 
Proof. Since $(S, T)$ is a barrier,

$$
\begin{aligned}
\delta(S, T) & =2|S|-2|T|+\sum_{y \in T} d_{G-S}(y)-h(S, T) \\
& =2|S|-2|T|+\sum_{y \in T} d_{G-S}(y)-\sum_{k \geqslant 0}\left|\mathcal{H}_{2 k+1}\right| \leqslant-2 .
\end{aligned}
$$

By Lemma $3.2(1)$ and (2),

$$
\sum_{y \in T} d_{G-S}(y)=\sum_{y \in T} e_{G}(y, U)=e_{G}(T, U)=\sum_{k \geqslant 0}(2 k+1)\left|\mathcal{H}_{2 k+1}\right| .
$$

Therefore, we have

$$
-2 \geqslant 2|S|-2|T|+\sum_{k \geqslant 0}(2 k+1)\left|\mathcal{H}_{2 k+1}\right|-\sum_{k \geqslant 0}\left|\mathcal{H}_{2 k+1}\right|,
$$

which yields $|T| \geqslant|S|+\sum_{k \geqslant 1} k\left|\mathcal{H}_{2 k+1}\right|+2$.

We perform the following operations to $G$.

(1) Remove all even components, and remove all components in $\mathcal{H}_{1}$.

(2) Remove all edges in $G[S]$.

(3) For a component $D \in \mathcal{H}_{2 k+1}$ with $k \geqslant 1$ introduce a set of $k$ independent vertices $U^{D}=\left\{u_{1}^{D}, u_{2}^{D}, \ldots, u_{k}^{D}\right\}$ and replace $D$ with $U^{D}$. By Lemma $3.2(3),\left|N_{G}(D) \cap T\right|=$ $e_{G}(T, D)=2 k+1$. Let $N_{G}(D) \cap T=\left\{v_{0}, v_{1}, \ldots, v_{2 k}\right\}$. Add two new edges $u_{i}^{D} v_{2 i-1}$ and $u_{i}^{D} v_{2 i}$ for each $i$ with $1 \leqslant i \leqslant k$. Moreover, add one extra edge $u_{1}^{D} v_{0}$.

Let $H$ be the resulting graph, and let

$$
U^{\mathcal{H}}=\bigcup_{k \geqslant 1}\left(\bigcup_{D \in \mathcal{H}_{2 k+1}} U^{D}\right), \quad X=S \cup U^{\mathcal{H}} .
$$

By the construction, the graph $H$ satisfies the following properties.

(1) $H$ is a bipartite graph with partite sets $X$ and $T$,

(2) $\left|U^{\mathcal{H}}\right|=\sum_{k \geqslant 1} k\left|\mathcal{H}_{2 k+1}\right|,|X|=|S|+\left|U^{\mathcal{H}}\right|=|S|+\sum_{k \geqslant 1} k\left|\mathcal{H}_{2 k+1}\right|$, and

(3) For each $k \geqslant 1$ and each $D \in \mathcal{H}_{2 k+1}, d_{H}\left(u_{1}^{D}\right)=3$ and $d_{H}\left(u_{i}^{D}\right)=2$ for each $i$ with $2 \leqslant i \leqslant k$.

We will show that there is a matching in $H$ which saturates $T$, which gives that $|X|=|S|+\sum_{k \geqslant 1} k\left|\mathcal{H}_{2 k+1}\right| \geqslant|T|$, giving a contradiction to Claim 4.1.

For notational simplicity, for a set $\mathcal{D} \subseteq \mathcal{H}$, let

$$
V(\mathcal{D})=\bigcup_{D \in \mathcal{D}} V(D)
$$


Claim 4.2. $|S|<\left|U^{\mathcal{H}}\right|$.

Proof. Assume to the contrary that $|S| \geqslant\left|U^{\mathcal{H}}\right|=\sum_{k \geqslant 1} k\left|\mathcal{H}_{2 k+1}\right|$. We may assume that $\left|U^{\mathcal{H}}\right| \geqslant 1$. For otherwise, since there is no edge between even component of $G-(S \cup T)$ and $T$, and each component in $\mathcal{H}_{1}$ is connected to a single vertex in $T, c(G-S) \geqslant|T|$. Since $|T| \geqslant 2$ by Claim 4.1,S is a cutset of $G$. This implies that $\tau(G) \leqslant \frac{|S|}{|T|}<1$, giving a contradiction.

For each $D \in \mathcal{H}_{2 k+1}$ with $k \geqslant 1$, let $W_{D}$ be a set of any $2 k$ vertices in $D$ such that for each $x \in W_{D}, e_{G}(x, T)=1$. Thus, $D-W_{D}$ is only connected to a single vertex in $T$. Let

$$
W=S \cup\left(\bigcup_{D \in \mathcal{H}_{2 k+1}, k \geqslant 1} W_{D}\right)
$$

Since $T$ is an independent set in $G$, and each component in $G-W$ is connected to $S$ or only a single vertex in $T$, we have that $c(G-W) \geqslant|T|$. Again, $W$ is a cutset of $G$ as $|T| \geqslant 2$ by Claim 4.1. So

$$
\begin{aligned}
\tau(G) & \leqslant \frac{|W|}{|T|} \leqslant \frac{|S|+\sum_{k \geqslant 1} 2 k\left|\mathcal{H}_{2 k+1}\right|}{|S|+\left|U^{\mathcal{H}}\right|+2} \\
& \leqslant \frac{\sum_{k \geqslant 1} k\left|\mathcal{H}_{2 k+1}\right|+\sum_{k \geqslant 1} 2 k\left|\mathcal{H}_{2 k+2}\right|}{\sum_{k \geqslant 1} k\left|\mathcal{H}_{2 k+1}\right|+\sum_{k \geqslant 1} k\left|\mathcal{H}_{2 k+1}\right|+2}<\frac{3}{2},
\end{aligned}
$$

showing a contradiction to the assumption that $\tau(G) \geqslant 3 / 2$.

Because of $|T|>|S|+\left|U^{\mathcal{H}}\right|$ and $\left|U^{\mathcal{H}}\right|>|S|$, we get the following Claim.

Claim 4.3. $|T| \geqslant 2|S|+2$.

Claim 4.4. $T$ contains no $\Delta$-vertex of $G$.

Proof. Suppose to the contrary that there exists $z \in T$ such that $d_{G}(z)=\Delta$. We may assume that $|\mathcal{H}(z)| \geqslant 2$. Otherwise, $e_{G}(z, S) \geqslant \Delta-1$ and so $|S| \geqslant \Delta-1$. Hence by Claims 4.2 and 4.3 ,

$$
\begin{aligned}
n & =|S|+|T|+|U| \\
& \geqslant 3|S|+2+\left|U^{\mathcal{H}}\right| \quad\left(|U| \geqslant\left|U^{\mathcal{H}}\right| \text { by Lemma } 3.2(4)\right) \\
& \geqslant 4|S|+3 \geqslant 4 \Delta-1 \geqslant 4 n / 3-1,
\end{aligned}
$$

implying that $n \leqslant 3$. This gives a contradiction to the fact that $\Delta \geqslant 3$.

Hence, by Lemma 3.3, we have that

$$
\begin{aligned}
n & =|S|+|T|+|U| \geqslant e_{G}(z, S)+3|\mathcal{H}(z)|+|T| \\
& \geqslant e_{G}(z, S)+3|\mathcal{H}(z)|+2 e_{G}(z, S)+2 \quad\left(|T| \geqslant 2|S|+2 \geqslant 2 e_{G}(z, S)+2\right) \\
& =3\left(e_{G}(z, S)+|\mathcal{H}(z)|\right)+2=3 \Delta+2 \geqslant n+2,
\end{aligned}
$$

showing a contradiction. 
Claim 4.5. $|\mathcal{H}| \geqslant 2$.

Proof. Assume to the contrary that $|\mathcal{H}| \leqslant 1$. By Claim 4.2, we then know that $|\mathcal{H}|=1$ and the component is adjacent to at least three vertices in $T$. Let $D$ be the only odd component in $G-(S \cup T)$ and assume that $e_{G}(D, T)=2 k+1$ for some $k \geqslant 1$. By Lemma $3.2(3),|T| \geqslant 2 k+1$. Again, by Claim $4.2,|S| \leqslant k-1$. Let $W$ be the union of the set $S$ and the set of neighbors of vertices in $T$ from the component $D$. Then $|W| \leqslant k-1+2 k+1=3 k$ and $c(G-W) \geqslant|T| \geqslant 2 k+1$. This gives a contradiction to the toughness of $G$.

Let $D_{1} \in \mathcal{H}$ be a component such that

$$
\left|V\left(D_{1}\right)\right|=\max \{|V(D)| \mid D \in \mathcal{H}\},
$$

and $D_{2} \in \mathcal{H}-\left\{D_{1}\right\}$ such that

$$
\left|V\left(D_{2}\right)\right|=\max \left\{|V(D)| \mid D \in \mathcal{H}-\left\{D_{1}\right\}\right\} .
$$

Claim 4.6. Let $D \in \mathcal{H}-\left\{D_{1}, D_{2}\right\}$. Then $D$ contains no $\Delta$-vertex of $G$. Furthermore, if $D_{1}$ contains a $\Delta$-vertex of $G$, then $|V(D)| \leqslant\left|V\left(D_{1}\right)\right|-1$; and if $D_{2}$ contains a $\Delta$-vertex of $G$, then $|V(D)| \leqslant\left|V\left(D_{2}\right)\right|-2 \leqslant\left|V\left(D_{1}\right)\right|-2$, and for any $x \in V(D), d_{G}(x) \leqslant \Delta-2$.

Proof. Note that by the choice of $D_{1}$ and $D_{2},|V(D)| \leqslant|V(\mathcal{H})| / 3$, recall here that $V(\mathcal{H})$ is the union of vertex sets of components in $\mathcal{H}$. Since $|T| \geqslant 2|S|+2$ by Claim 4.3 , we have that $n \geqslant|S|+|T|+|V(\mathcal{H})| \geqslant 3|S|+2+|V(\mathcal{H})|$. Consequently, $|V(\mathcal{H})| / 3 \leqslant$ $(n-2) / 3-|S|$. Thus, for any $x \in V(D)$,

$$
d_{G}(x) \leqslant|V(D)|-1+1+|S| \leqslant|V(\mathcal{H})| / 3+|S| \leqslant(n-2) / 3<\Delta .
$$

Suppose that $D_{1}$ contains a $\Delta$-vertex of $G$, and there exists $D \in \mathcal{H}-\left\{D_{1}, D_{2}\right\}$ such that $|V(D)|=\left|V\left(D_{1}\right)\right|$. This implies that $\left|V\left(D_{1}\right)\right|=\left|V\left(D_{2}\right)\right|=|V(D)|$, so $\left|V\left(D_{1}\right)\right| \leqslant$ $|V(\mathcal{H})| / 3$. Then by exactly the same argument above, we have that for any $x \in V\left(D_{1}\right)$,

$$
d_{G}(x) \leqslant|V(D)|-1+1+|S| \leqslant|V(\mathcal{H})| / 3+|S| \leqslant(n-2) / 3<\Delta .
$$

Hence, $|V(D)| \leqslant\left|V\left(D_{1}\right)\right|-1$.

Suppose now that $D_{2}$ contains a $\Delta$-vertex of $G$. Since $\left|V\left(D_{1}\right)\right| \geqslant\left|V\left(D_{2}\right)\right|$, we then have that $\left|V\left(D_{i}\right)\right|+|S| \geqslant \Delta$ for $i=1,2$. So for any $D \in \mathcal{H}-\left\{D_{1}, D_{2}\right\}$,

$$
\begin{aligned}
n & \geqslant|S|+|T|+\left|V\left(D_{1}\right)\right|+\left|V\left(D_{2}\right)\right|+|V(D)| \\
& \geqslant|S|+2|S|+2+\left|V\left(D_{1}\right)\right|+\left|V\left(D_{2}\right)\right|+|V(D)| \\
& =|S|+\left|V\left(D_{1}\right)\right|+|S|+\left|V\left(D_{2}\right)\right|+|S|+|V(D)|+2 .
\end{aligned}
$$

Because of $\left|V\left(D_{i}\right)\right|+|S| \geqslant \Delta$ for $i=1,2$, it follows that

$$
|S|+|V(D)| \leqslant n-2 \Delta-2 \leqslant n / 3-2 \leqslant \Delta-2 .
$$


Consequently, $|V(D)| \leqslant\left|V\left(D_{2}\right)\right|-2 \leqslant\left|V\left(D_{1}\right)\right|-2$, and for any $x \in V(D), d_{G}(x) \leqslant$ $\Delta-2$.

We introduce some further notation here. Let

$$
T_{1}=\left\{y \in T|| \mathcal{H}_{1}(y) \mid=1\right\}, \quad \text { and } \quad T_{2}=\left\{y \in T|| \mathcal{H}_{1}(y) \mid \geqslant 2\right\} .
$$

For each component $D \in \mathcal{H}_{1}$, let $y_{D} \in T$ be the vertex such that $e_{G}(D, T)=e_{G}\left(D, y_{D}\right)=$ 1. Let

$$
\mathcal{H}_{11}=\left\{D \in \mathcal{H}_{1} \mid y_{D} \in T_{1}\right\}, \quad \text { and } \quad \mathcal{H}_{12}=\left\{D \in \mathcal{H}_{1} \mid y_{D} \in T_{2}\right\}\left(=\mathcal{H}_{1}-\mathcal{H}_{11}\right) .
$$

Claim 4.7. For each component $D \in \mathcal{H}_{12},\left|\mathcal{H}\left(y_{D}\right)\right| \geqslant 2$. Consequently, $|V(D)| \geqslant 3$.

Proof. Since $D \in \mathcal{H}_{12}$, we have that $\left|\mathcal{H}\left(y_{D}\right)\right| \geqslant\left|\mathcal{H}_{1}\left(y_{D}\right)\right| \geqslant 2$. Then $|V(D)| \geqslant 3$ by Lemma 3.3.

Denote

$$
\begin{aligned}
m_{1} & =\left|\mathcal{H}_{11}\right|, \quad m_{2}=\left|\mathcal{H}_{12}\right|, \quad \text { and } \quad m_{3}=\left|\mathcal{H}-\mathcal{H}_{1}\right|, \\
S_{1} & =\{x \in S \mid x \text { has a non } \Delta \text {-degree neighbor in } V(G)-T\}, \quad \text { and } S_{0}=S-S_{1}, \\
p_{y} & =\left|\mathcal{H}_{1}(y)\right| \quad \text { for any } y \in T .
\end{aligned}
$$

Note that by the definition, if $m_{2} \neq 0$, then $m_{2} \geqslant 2$.

Claim 4.8. Let $y \in T$ be a vertex. Then

$$
\left|N_{G}(y) \cap S\right| \geqslant \begin{cases}\left|S_{0}\right|+m_{1} / 3+m_{2}-1, & \text { if } \emptyset \neq \mathcal{H}(y) \nsubseteq\left\{D_{1}, D_{2}\right\} \\ 2, & \text { if } \mathcal{H}(y)=\left\{D_{1}\right\} \text { or }\left\{D_{2}\right\} \\ 1, & \text { if } \mathcal{H}(y)=\left\{D_{1}, D_{2}\right\}\end{cases}
$$

Moreover, $N_{G}(y) \cap S \neq \emptyset$.

Proof. Since $G$ is $3 / 2$-tough, $\delta(G) \geqslant 3$. As each $y \in T_{1}$ satisfies $d_{G}(y)=e_{G}(y, S)+$ $e_{G}(y, V(\mathcal{H}))$ and $e_{G}\left(y, D_{i}\right) \leqslant 1$ for $i=1,2$, we get $e_{G}(y, S) \geqslant 2$ if $\mathcal{H}(y)=\left\{D_{1}\right\}$ or $\left\{D_{2}\right\}$. If $\mathcal{H}(y)=\left\{D_{1}, D_{2}\right\}$, then $\left|N_{G}(y) \cap S\right| \geqslant 1$.

Thus we assume that there exists $D \in \mathcal{H}(y)-\left\{D_{1}, D_{2}\right\}$. Let $x_{D}$ be the neighbor of $y$ in $D$. By Claim 4.6, $x_{D}$ is not a $\Delta$-vertex of $G$. Moreover, $y$ is adjacent to at least $\Delta-d_{G}\left(x_{D}\right)+1 \Delta$-vertices of $G$ by Vizing's Adjacency Lemma.

Note that each component in $\mathcal{H}-\mathcal{H}_{1}$ contains at least three vertices by Lemma 3.2 (4). So $n \geqslant$

$$
\begin{cases}|S|+|T|+\left|V\left(D_{1}\right)\right|+\left|V\left(D_{2}\right)\right|+|V(D)|+m_{1}+3\left(m_{2}+m_{3}-3\right), & \text { if } D_{1}, D_{2}, D \\ |S|+|T|+\left|V\left(D_{1}\right)\right|+\left|V\left(D_{2}\right)\right|+|V(D)|+m_{1}-1+3\left(m_{2}+m_{3}-2\right), & \text { otherwise. }\end{cases}
$$

Thus, because $|T| \geqslant 2|S|+2$ by Lemma 4.3 , and $\left|U^{\mathcal{H}}\right| \geqslant|S|+1$ implying that $m_{3} \geqslant 1$, we get that

$$
n \geqslant|S|+|T|+\left|V\left(D_{1}\right)\right|+\left|V\left(D_{2}\right)\right|+|V(D)|+m_{1}+3\left(m_{2}+m_{3}-3\right)
$$




$$
\begin{aligned}
& \geqslant 3|S|+2+\left|V\left(D_{1}\right)\right|+\left|V\left(D_{2}\right)\right|+|V(D)|+m_{1}+3\left(m_{2}-2\right) \\
& \geqslant \begin{cases}3|S|+3|V(D)|+m_{1}+3 m_{2}, & \text { if } D_{2} \text { contains a } \Delta \text {-vertex; } \\
3|S|+3|V(D)|+m_{1}+3 m_{2}-3, & \text { if } D_{1} \text { contains a } \Delta \text {-vertex; } \\
3|S|+3|V(D)|+m_{1}+3 m_{2}-4, & \text { if neither } D_{1} \text { nor } D_{2} \text { contains a } \Delta \text {-vertex. }\end{cases}
\end{aligned}
$$

The above bounds were obtained because of $\left|V\left(D_{1}\right)\right| \geqslant\left|V\left(D_{2}\right)\right| \geqslant|V(D)|$ and Claim 4.6. Thus since no component in $\mathcal{H}-\left\{D_{1}, D_{2}\right\}$ containing a $\Delta$-vertex of $G$ by Claim 4.6, we have that

$$
\left|N_{G}(y) \cap S \cap V_{\Delta}\right| \geqslant \begin{cases}\Delta-d_{G}\left(x_{D}\right)-1, & \text { if } D_{2} \text { contains a } \Delta \text {-vertex; } \\ \Delta-d_{G}\left(x_{D}\right), & \text { if } D_{1} \text { contains a } \Delta \text {-vertex but } D_{2} \text { has no } \Delta \text {-vertex; } \\ \Delta-d_{G}\left(x_{D}\right)+1, & \text { if neither } D_{1} \text { nor } D_{2} \text { contains a } \Delta \text {-vertex. }\end{cases}
$$

Because $x_{D}$ is not a $\Delta$-vertex of $G$, by the definitions of $S_{0}$ and $S_{1}$, we have that $N_{G}\left(x_{D}\right) \cap$ $S=N_{G}\left(x_{D}\right) \cap S_{1}$. So $d_{G}\left(x_{D}\right) \leqslant\left|S_{1}\right|+|V(D)|$. Replacing $\Delta$ by $\frac{n}{3}$ in the above bounds on $\left|N_{G}(y) \cap S\right|$, and combining the bounds on $n$, we get that

$$
\left|N_{G}(y) \cap S\right| \geqslant \begin{cases}\left|S_{0}\right|+\frac{m_{1}}{3}+m_{2}-1, & \text { if } D_{2} \text { contains a } \Delta \text {-vertex; } \\ \left|S_{0}\right|+\frac{m_{1}}{3}+m_{2}-1, & \text { if } D_{1} \text { contains a } \Delta \text {-vertex but } D_{2} \text { has no } \Delta \text {-vertex; } \\ \left|S_{0}\right|+\frac{m_{1}}{3}+m_{2}-\frac{1}{3}, & \text { if neither } D_{1} \text { nor } D_{2} \text { contains a } \Delta \text {-vertex. }\end{cases}
$$

For the second part of the statement, if $\mathcal{H}(y)=\emptyset$, then $N_{G}(y)=N_{G}(y) \cap S$. So assume that $\mathcal{H}(y) \neq \emptyset$. By the first part of the statement, it easily follows that $\left|N_{G}(y) \cap S\right| \geqslant 1$ unless $\mathcal{H}(y) \nsubseteq\left\{D_{1}, D_{2}\right\}$. Let $D \in \mathcal{H}(y)-\left\{D_{1}, D_{2}\right\}$, and let $x_{D}$ be the neighbor of $y$ in $D$. By Claim 4.6, $x_{D}$ is not a $\Delta$-vertex of $G$. Moreover, $y$ is adjacent to at least $\Delta-d_{G}\left(x_{D}\right)+1$ $\Delta$-vertices of $G$ by Vizing's Adjacency Lemma. Note that no component in $\mathcal{H}-\left\{D_{1}, D_{2}\right\}$ contains a $\Delta$-vertex of $G$ by Claim 4.6. If $D_{2}$ does not contain a $\Delta$-vertex of $G$, then $y$ is adjacent to at least $\Delta-d_{G}\left(x_{D}\right) \geqslant 1 \Delta$-vertices which are contained in $S$. If $D_{2}$ contains a $\Delta$-vertex of $G$, then by the second part of Claim $4.6, d_{G}\left(x_{D}\right) \leqslant \Delta-2$. So $y$ is adjacent to at least $\Delta-d_{G}\left(x_{D}\right)-1 \geqslant 1 \Delta$-vertices which are contained in $S$.

The proof is finished.

If $\left\{D_{1}, D_{2}\right\} \cap \mathcal{H}_{1} \neq \emptyset$, say $D_{1} \in \mathcal{H}_{1}$, then there exists a unique vertex $y \in T$ such that $e_{G}\left(y, D_{1}\right)=1$. If $\left\{D_{1}, D_{2}\right\} \cap \mathcal{H}_{1} \neq \emptyset$ and there exists $y \in T$ such that $\mathcal{H}(y)=\left\{D_{1}, D_{2}\right\}$,

$$
\text { we denote } y \text { by } y_{\omega} \text {. }
$$

Claim 4.9. Let $y \in T$ be a vertex such that $|\mathcal{H}(y)| \geqslant 2$ and $y \neq y_{\omega}$. Then $d_{G}(y) \geqslant 4$.

Proof. Assume to the contrary that $d_{G}(y)=3$. Let $D \in \mathcal{H}(y)-\left\{D_{1}, D_{2}\right\}$, and let $x_{D}$ be the neighbor of $y$ in $D$. Then $x_{D}$ is adjacent to at least $\Delta-3+1 \Delta$-vertices of $G$ by Vizing's Adjacency Lemma. Since $V(D)$ contains no $\Delta$-vertex of $G$ by Claim 4.6, and $T$ contains no $\Delta$-vertex of $G$ by Claim 4.4, we conclude that $|S| \geqslant\left|N_{G}\left(x_{D}\right) \cap S \cap V_{\Delta}\right| \geqslant \Delta-2$. Since each $D \in \mathcal{H}_{2 k+1}$ contains at least $2 k+1$ vertices by Lemma $3.2(4),|V(\mathcal{H})| \geqslant 2\left|U^{\mathcal{H}}\right|$. Thus

$$
n \geqslant|S|+2\left|U^{\mathcal{H}}\right|+|T| \geqslant|S|+2(|S|+1)+2|S|+2
$$




$$
=5|S|+4 \geqslant 5(\Delta-2)+4 \geqslant 5\left(\frac{n}{3}-2\right)+4=\frac{5 n}{3}-6,
$$

implying that $n \leqslant 9$.

By Claim 4.8, $N_{G}(y) \cap S \neq \emptyset$. Since $|\mathcal{H}(y)| \geqslant 2$, by Lemma 3.3, $|U| \geqslant|V(\mathcal{H}(y))| \geqslant 6$. Since $|S| \geqslant 1,|T| \geqslant 2|S|+2 \geqslant 4$. Hence, $n \geqslant|S|+|T|+|U| \geqslant 1+4+6 \geqslant 11$, a contradiction.

Claim 4.10. Let $x y \in E(H)$ be an edge with $x \in X$ and $y \in T$. Then each of the following holds.

(1) If $x \in S_{0}$, then $d_{H}(y)+p_{y} \geqslant d_{H}(x)+1$.

(2) If $x \in S_{1}$, then $d_{H}(y)+p_{y} \geqslant d_{H}(x)+2$.

(3) If $x \in U^{\mathcal{H}}$ and $p_{y}=0$, then $d_{H}(y) \geqslant d_{H}(x)$.

(4) If $x \in U^{\mathcal{H}}, p_{y} \geqslant 1$, and $y \neq y_{\omega}$, then $d_{H}(y)+p_{y} \geqslant d_{H}(x)+1$.

(5) If $x \in U^{\mathcal{H}}$ and $y=y_{\omega}$, then $d_{H}(y)+p_{y} \geqslant d_{H}(x)$.

Proof. Statements (1) and (2) follow from Lemma 2.2 by taking $\sigma_{x}=0$ and 1, respectively. The statements (3) and (5) are clear, since $d_{H}(y)+p_{y}=d_{G}(y) \geqslant \delta(G) \geqslant 3$, and $d_{H}(x) \leqslant 3$ for any $x \in U^{\mathcal{H}}$. Now we show statement (4). By the assumption that $x \in U^{\mathcal{H}}$ and $p_{y} \geqslant 1$, we have that $|\mathcal{H}(y)| \geqslant 2$. Then the statement follows by Claim 4.9, since $d_{H}(y)+p_{y}=d_{G}(y) \geqslant 4$, while $d_{H}(x) \leqslant 3$.

Claim 4.11. $H$ has a matching which saturates $T$.

Proof. Suppose to the contrary that $H$ has no matching saturating $T$. By Hall's Theorem, there is a nonempty subset $B \subseteq T$ such that $\left|N_{H}(B)\right|<|B|$. Among all such subsets with this property, we choose $B$ with smallest cardinality. Let $A=N_{H}(B)$ and $H^{\prime}=H[A \cup B]$. Then we claim that in $H^{\prime}$, there is a matching which saturates $A$. Suppose this is not the case. By Hall's Theorem again, there is a nonempty subset $A^{\prime} \subseteq A$ such that $\left|N_{H^{\prime}}\left(A^{\prime}\right)\right|<\left|A^{\prime}\right|$. Since $A^{\prime} \subseteq A=N_{H}(B) \neq \emptyset(T$ contains no isolated vertex of $H), N_{H^{\prime}}\left(A^{\prime}\right) \neq \emptyset$. Let $B^{\prime}=B-N_{H^{\prime}}\left(A^{\prime}\right)$. As $|B|>|A| \geqslant\left|N_{H^{\prime}}\left(A^{\prime}\right)\right|>0$, $0<\left|B^{\prime}\right|<|B|$. On the other hand, we have $N_{H^{\prime}}\left(B^{\prime}\right)=N_{H}\left(B^{\prime}\right) \subseteq A-A^{\prime}$. However, $\left|B^{\prime}\right|=|B|-\left|N_{H^{\prime}}\left(A^{\prime}\right)\right|>|A|-\left|N_{H^{\prime}}\left(A^{\prime}\right)\right|>|A|-\left|A^{\prime}\right|=\left|A-A^{\prime}\right| \geqslant\left|N_{H}\left(B^{\prime}\right)\right|$, showing a contradiction to the choice of $B$.

Let $M$ be a matching of $H^{\prime}=H[A \cup B]$ which saturates $A$. We consider two cases below.

Case 1. $B \cap T_{2} \subseteq\left\{y_{\omega}\right\}$.

For any $y \in B$ with $y \neq y_{\omega}, p_{y} \leqslant 1$. Since $|B|>|A|$, there exists $y_{0} \in B-V(M)$. Since $p_{y_{0}} \leqslant 1$ if $y_{0} \neq y_{\omega}$, we have that $d_{H}\left(y_{0}\right) \geqslant 2$ if $y_{0} \neq y_{\omega}$. Otherwise, $d_{H}\left(y_{0}\right) \geqslant 1$.

Assume first that $y_{\omega} \notin V(M)$. So applying Claim 4.10, we have that

$$
e_{H}(A, B) \leqslant \sum_{\substack{x y \in M \\ x \in A, y \in B}} d_{H}(x)
$$




$$
\begin{aligned}
& \leqslant \sum_{\substack{x y \in M \\
x \in S_{0} \cup S_{1} \text { Or } \\
p_{y}=1}}\left(d_{H}(y)+p_{y}-1\right)+\sum_{\substack{x y \in M \\
x \in U}, y \in B, p_{y}=0} d_{H}(y) \\
& <\sum_{\substack{x y \in M \\
x \in A, y \in B}} d_{H}(y)+d_{H}\left(y_{0}\right) \leqslant e_{H}(A, B),
\end{aligned}
$$

showing a contradiction.

Assume now that $y_{\omega} \in V(M)$. By the definition of $y_{\omega}, 1 \leqslant p_{y_{\omega}} \leqslant 2$. If $p_{y_{\omega}}=2$, then for any edge $x y_{\omega} \in E(H)$, we have that $x \in S$ and so $d_{H}\left(y_{\omega}\right)+p_{y_{\omega}} \geqslant d_{H}(x)+1$; and if $p_{y_{\omega}}=1$, then for any edge $x y_{\omega} \in E(H), d_{H}\left(y_{\omega}\right)+p_{y_{\omega}} \geqslant d_{H}(x)$. So for any edge $x y_{\omega} \in E(H), d_{H}(x) \leqslant d_{H}\left(y_{\omega}\right)+1$. Then applying Claim 4.10, we have that

$$
\begin{aligned}
& e_{H}(A, B) \leqslant \sum_{\substack{x y \in M \\
x \in A, y \in B}} d_{H}(x) \\
\leqslant & \sum_{\substack{x y \in M \\
x \in S_{0} \cup S_{1} \text { Or } p_{y}=1, y \neq y_{\omega}}}\left(d_{H}(y)+p_{y}-1\right)+\left(\sum_{\substack{x y \in M \\
x \in U \mathcal{H}, y \in B, p_{y}=0}} d_{H}(y)\right)+d_{H}\left(y_{\omega}\right)+1 \\
< & \left(\sum_{\substack{x y \in M \\
x \in A, y \in B}} d_{H}(y)\right)+d_{H}\left(y_{0}\right) \leqslant e_{H}(A, B),
\end{aligned}
$$

showing a contradiction again.

Case 2. $\left(B \cap T_{2}\right)-\left\{y_{\omega}\right\} \neq \emptyset$.

For any $y \in T_{2}, \mathcal{H}_{1}(y) \subseteq \mathcal{H}_{12}$. Since $\left|\mathcal{H}_{1}(y)\right| \geqslant 2$ if $y \in T_{2}$, the assumption that $\left(B \cap T_{2}\right)-\left\{y_{\omega}\right\} \neq \emptyset$ implies that $m_{2} \geqslant 2$. Furthermore, if $y_{\omega} \in T_{2}$, then $m_{2} \geqslant 4$.

Since $|B|>|A|$, there exists $y_{0} \in B-V(M)$. Since $N_{H}(y) \cap S \neq \emptyset$ for any $y \in T$ by Claim 4.8, we have $d_{H}\left(y_{0}\right) \geqslant 1$. We claim that if $y_{\omega}$ exists and $y_{0} \neq y_{\omega}$, then $d_{H}\left(y_{0}\right) \geqslant 2$. If $\left|\mathcal{H}\left(y_{0}\right)\right| \leqslant 1$, then $d_{H}\left(y_{0}\right) \geqslant d_{G}\left(y_{0}\right)-1 \geqslant 2$. So we assume that $\left|\mathcal{H}\left(y_{0}\right)\right| \geqslant 2$. If $y_{\omega}$ exists and $y_{0} \neq y_{\omega}$, then by Claim 4.8, $d_{H}\left(y_{0}\right) \geqslant\left|N_{H}\left(y_{0}\right) \cap S\right| \geqslant\left|S_{0}\right|+m_{1} / 3+m_{2}-1 \geqslant m_{1} / 3+m_{2}-1$. Note that if $y_{\omega} \in T_{2}$ then $m_{2} \geqslant 4$, and if $y_{\omega} \notin T_{2}$, then by the definition of $y_{\omega}, m_{1} \geqslant 1$. Thus we have that $d_{H}\left(y_{0}\right) \geqslant 2$.

For any $y \in T_{2}-\left\{y_{\omega}\right\},\left|N_{H}(y) \cap S\right|=\left|N_{G}(y) \cap S\right| \geqslant\left|S_{0}\right|+m_{1} / 3+m_{2}-1$ by Claim 4.8. Thus, $|A \cap S| \geqslant\left|S_{0}\right|+m_{1} / 3+m_{2}-1$. Let $A_{0}=A \cap S$. Then since $m_{2} \geqslant 2$, if $m_{1} \leqslant 1$

$$
2\left|A_{0}-S_{0}\right| \geqslant 2 m_{1} / 3+2 m_{2}-2 \geqslant m_{1}+m_{2}-1 / 3,
$$

and if $m_{1} \geqslant 2$, then

$$
\left|A_{0}-S_{0}\right| \geqslant m_{1} / 3+m_{2}-1 \geqslant m_{2}-1 / 3 .
$$

If $y_{\omega} \in V(M)$, then let $x_{\omega}$ be the vertex with $x_{\omega} y_{\omega} \in M$.

Assume first that $y_{\omega} \notin V(M)$ or $x_{\omega} \in S$. Note that in both cases, Claim 4.10 (5) does not apply. Applying Claim 4.10 (1-4), we have that

$$
e_{H}(A, B) \leqslant \sum_{\substack{x y \in M \\ x \in A, y \in B}} d_{H}(x) \leqslant \sum_{\substack{x y \in M \\ x \in S_{0}}}\left(d_{H}(y)+p_{y}-1\right)+\sum_{\substack{x y \in M \\ x \in S_{1}}}\left(d_{H}(y)+p_{y}-2\right)
$$




$$
\begin{aligned}
& +\sum_{\substack{x y \in M \\
x \in U}, y \in B, p_{y}=0} d_{H}(y)+\sum_{\substack{x y \in M \\
x \in U \mathcal{H}, y \in B, p_{y} \geqslant 1}}\left(d_{H}(y)+p_{y}-1\right) \\
& \leqslant\left\{\begin{array}{l}
\left(\sum_{\substack{x y \in M \\
x \in A, y \in B}} d_{H}(y)\right)+\sum_{\substack{x y \in M \\
x \notin S_{1}}} p_{y}+\sum_{\substack{x y \in M \\
x \in S_{1}}}\left(p_{y}-2\right) \\
\left(\sum_{\substack{x y \in M \\
x \in A, y \in B}} d_{H}(y)\right)+\sum_{\substack{x y \in M \\
p_{y}=1}}\left(p_{y}-1\right)+\sum_{\substack{x y \in M \\
x \in S_{1} \text { or }}}\left(p_{y} \geqslant 2\right.
\end{array}\right. \\
& \leqslant\left\{\begin{array}{l}
\left(\sum_{\substack{x \in M \\
x \in A, y \in B}} d_{H}(y)\right)+m_{1}+m_{2}-2\left|A_{0}-S_{0}\right| \\
\left.\sum_{\substack{x y \in M \\
x \in A, y \in B}} d_{H}(y)\right)+m_{2}-\left|A_{0}-S_{0}\right|
\end{array}\right. \\
& \leqslant\left(\sum_{\substack{x \in M M \\
x \in A, y \in B}} d_{H}(y)\right)+1 / 3 \\
& <\left(\sum_{\substack{x y \in M \\
x \in A, y \in B}} d_{H}(y)\right)+d_{H}\left(y_{0}\right) \leqslant e_{H}(A, B),
\end{aligned}
$$

showing a contradiction.

Assume now that $y_{\omega} \in V(M)$ and $x_{\omega} \in U^{\mathcal{H}}$. By the definition of $y_{\omega}, 1 \leqslant p_{y_{\omega}} \leqslant 2$. If $p_{y_{\omega}}=2$, then since $d_{H}\left(y_{\omega}\right) \geqslant p_{y_{\omega}}=2$, we have $d_{H}\left(y_{\omega}\right)+p_{y_{\omega}} \geqslant 4 \geqslant d_{H}(x)+1$ for each edge $x y_{\omega} \in E(H)$ with $x \in U^{\mathcal{H}}$; and if $p_{y_{\omega}}=1$, then for any edge $x y_{\omega} \in E(H)$, $d_{H}\left(y_{\omega}\right)+p_{y_{\omega}} \geqslant d_{H}(x)$. Therefore, combining Claim 4.10, we know that for any edge $x y_{\omega} \in E(H), d_{H}(x) \leqslant d_{H}\left(y_{\omega}\right)+1$. Applying Claim 4.10 again, we have that

$$
\begin{aligned}
& e_{H}(A, B) \leqslant \sum_{\substack{x y \in M \\
x \in A, y \in B}} d_{H}(x) \leqslant \sum_{\substack{x y \in M \\
x \in S_{0}}}\left(d_{H}(y)+p_{y}-1\right)+\sum_{\substack{x y \in M \\
x \in S_{1}}}\left(d_{H}(y)+p_{y}-2\right) \\
& +\sum_{\substack{x y \in M \\
x \in U \mathcal{H}, y \in B, p_{y}=0}} d_{H}(y)+\sum_{\substack{x y \in M \\
x \in U \mathcal{H}, y \in B, p_{y} \geqslant 1, y \neq y_{\omega}}}\left(d_{H}(y)+p_{y}-1\right)+d_{H}\left(y_{\omega}\right)+1 \\
& \leqslant\left\{\begin{array}{l}
\left(\sum_{\substack{x y \in M \\
x \in A, y \in B}} d_{H}(y)\right)+\sum_{\substack{x y \in M \\
x \notin S_{1}}} p_{y}+\sum_{\substack{x y \in M \\
x \in S_{1}}}\left(p_{y}-2\right)+1 \\
\left(\sum_{\substack{x y \in M \\
x \in A, y \in B}} d_{H}(y)\right)+\sum_{\substack{x y \in M \\
p_{y}=1}}\left(p_{y}-1\right)+\sum_{\substack{x y \in M \\
x \in S_{1} \text { Or } \\
p_{y} \geqslant 2}}\left(p_{y}-1\right)+1
\end{array}\right.
\end{aligned}
$$




$$
\begin{aligned}
& \qquad\left\{\begin{array}{l}
\left(\sum_{\substack{x y \in M \\
x \in A, y \in B}} d_{H}(y)\right)+1+m_{1}+m_{2}-2\left|A_{0}-S_{0}\right| \\
\left.\leqslant \sum_{\substack{x y \in M \\
x \in A, y \in B}} d_{H}(y)\right)+1+m_{2}-\left|A_{0}-S_{0}\right|
\end{array}\right. \\
& \leqslant\left(\sum_{\substack{x y \in M \\
x \in A, y \in B}} d_{H}(y)\right)+1+1 / 3 \\
& <\left(\sum_{\substack{x y \in M \\
x \in A, y \in B}} d_{H}(y)\right)+d_{H}\left(y_{0}\right) \leqslant e_{H}(A, B), \quad\left(d_{H}\left(y_{0}\right) \geqslant 2 \text { in this case }\right)
\end{aligned}
$$

showing a contradiction again.

Claim 4.11 gives a contradiction to Claim 4.1. The proof of Theorem 1.3 is now complete.

\section{$5 \quad$ Essentiality of $\Delta$-criticality}

In this section, we construct a family of $n$-vertex graphs $G$ with $\Delta(G)=n-1, \tau(G) \geqslant \frac{3}{2}$, but have no 2 -factor. This demonstrates that the condition of $\Delta$-criticality cannot be dropped from Theorem 1.3.

Let $k \geqslant 7$ be an integer, $S$ be the vertex set of a complete graph of order $k, T$ be a set of $6 k+1$ isolated vertices, and $\mathcal{H}$ be a set of 5 disjoint complete graphs $Q_{1}, Q_{2}, Q_{3}, Q_{4}, Q_{5}$, each with order $2 k+1$. Label the vertices in $T$ as $u_{1}, u_{2}, \cdots, u_{6 k+1}$. Let $G$ be a graph with $V(G)=S \cup T \cup V(\mathcal{H})$. The edges of $G$ are constructed below:

Step 1 Adding all edges between $S$ and $T \cup V(\mathcal{H})$. (Clearly, every vertex in $S$ is adjacent to every other vertex of $G$. )

Step 2 For each $i, i=1,2,3,4$, joining each of

$$
u_{(i-1) \times(k-1)+1}, u_{(i-1) \times(k-1)+2}, \cdots, u_{(i-1) \times(k-1)+k-1}
$$

to one unused vertex in $Q_{i}$ and one unused vertex in $Q_{i+1}$; joining each of

$$
u_{4 k-3}, u_{4 k-2}, u_{4 k-1}, u_{4 k}, u_{4 k+1}, u_{4 k+2}, u_{4 k+3}, u_{4 k+4}
$$

to one unused vertex in $Q_{1}$ and one unused vertex in $Q_{5}$. (By "unused," we meant that the vertex in $Q_{i}$ has not been joined to any vertex in $T$. )

Step 3 After Step 2, each of $Q_{2}, Q_{3}$, and $Q_{4}$ has only 3 unused vertex left, and each of $Q_{1}$ and $Q_{5}$ has exactly $k-6$ unused vertex left. Also, there are exactly $(6 k+1)-$ $(4 k+4)=2 k-3$ vertices in $T$ that are not joined to any vertex in $\mathcal{H}$. Joining each vertex of $u_{4 k+5}, u_{4 k+6}, \cdots, u_{6 k+1}$ to exactly one unused vertex in $\mathcal{H}$. 
By the construction,

$$
n:=|V(G)|=k+5(2 k+1)+6 k+1=17 k+6 .
$$

All vertices in $S$ have degree $n-1$, which are the only maximum degree vertices in $G$. Each vertex in $T$ is adjacent to a vertex in $\mathcal{H}$ that has degree $2 k+1+|S|=3 k+1$, and is adjacent to exactly $k$ vertices of maximum degree. Since $n=17 k+6$, and

$$
\Delta(G)-(3 k+1)+1=n-1-3 k=14 k+5>k,
$$

Lemma 2.1 implies that $G$ is not $\Delta$-critical.

We next claim that $G$ has no 2 -factor. This is clear, since every $Q_{i}$ is an odd component of $G-(S \cup T)$ that is adjacent to exactly $2 k+1$ vertices in $T$ by the construction, and we have that

$$
\delta(S, T)=2 k-2 \times(6 k+1)+5 \times(2 k+1)-5=-2<0 .
$$

Thus, $(S, T)$ is a barrier and $G$ has no 2-factor by Lemma 3.1.

Finally, we show that $\tau(G) \geqslant \frac{3}{2}$. Let $W \subseteq V(G)$ be a toughset of $G$. That is, $W$ is a cutset of $G$ such that $\frac{|W|}{c(G-W)}=\tau(G)$. Assume to the contrary that $\tau(G)<\frac{3}{2}$. Since $G$ is 3-connected, the assumption that $\tau(G)<\frac{3}{2}$ implies that $c(G-W) \geqslant 3$.

Since every vertex in $S$ is adjacent to every other vertex in $G$, we get the following conclusion.

Claim 5.1. $S \subseteq W$.

Claim 5.2. Let $U \subseteq W$ be a proper non-empty subset of $W$. Then all vertices in $U$ are adjacent to in total at least $\frac{2|U|}{3}+1$ components of $G-W$. In particular, for any $x \in W$, $x$ is adjacent to at least two components of $G-W$.

Proof. Assume to the contrary that there exists a proper non-empty subset $U$ of $W$ such that all vertices in $U$ are adjacent to in total at most $\frac{2|U|}{3}$ components of $G-W$. Let $\tau=\tau(G)$. Then $|W|=\tau c(G-W)$ and $G-(W-U)$ has at least $c(G-W)-\frac{2|U|}{3}+1>$ $c(G-W)-\frac{|U|}{\tau}+1$ components of $G-W$. Since $\tau(G)<\frac{3}{2}$, we see that $c(G-(W-U)) \geqslant 2$. Thus $W^{*}=W-U$ is a cutset of $G$. However,

$$
\frac{\left|W^{*}\right|}{c\left(G-W^{*}\right)}<\frac{|W-U|}{c(G-W)-\frac{1}{\tau}|U|}=\tau,
$$

contradicting the fact that $W$ is a toughset of $G$.

Claim 5.3. $W \cap T=\emptyset$.

Proof. Assume to the contrary that there exists $x \in W \cap T$. Note that each vertex in $T$, besides being adjacent to all vertices in $S$, is adjacent to at most two vertices in $\mathcal{H}$. As $S \subseteq W$ by Claim 5.1, we know by the particular part of Claim 5.2 that $x \in$ 
$\left\{u_{1}, u_{2}, \cdots, u_{4 k+4}\right\}$ and the two neighbors of $x$ in $\mathcal{H}$ are contained in two distinct components of $G-W$. Assume that the neighbors of $x$ in $\mathcal{H}$ are in $Q_{1}$ and $Q_{5}$. (The argument for the other cases is similar.) Then $x \in\left\{u_{4 k-3}, u_{4 k-2}, u_{4 k-1}, u_{4 k}, u_{4 k+1}, u_{4 k+2}, u_{4 k+3}, u_{4 k+4}\right\}$. Let $W^{*}=W \cap\left(\left\{u_{4 k-3}, u_{4 k-2}, u_{4 k-1}, u_{4 k}, u_{4 k+1}, u_{4 k+2}, u_{4 k+3}, u_{4 k+4}\right\} \cup V\left(Q_{1} \cup Q_{5}\right)\right)$. It follows that $W^{*}$ separates the two neighbors of $x$ in $Q_{1} \cup Q_{5}$ into two distinct components in $G-W$. By the construction of the edges of $G$ in Step 2, we know that there are 8 vertex-disjoint paths between $Q_{1}$ and $Q_{5}$ going through the eight vertices $u_{4 k-3}, u_{4 k-2}, u_{4 k-1}, u_{4 k}, u_{4 k+1}, u_{4 k+2}, u_{4 k+3}, u_{4 k+4}$. Thus, $\left|W^{*}\right| \geqslant 8$. Note that each of $u_{4 k-3}, \cdots, u_{4 k+4}$ is only adjacent to $Q_{1}$ and $Q_{5}$ in $G-S$, and $Q_{1}$ and $Q_{5}$ are cliques in $G$. Assume there are exactly $t$ vertices $y$ from

$$
\left\{u_{4 k-3}, u_{4 k-2}, u_{4 k-1}, u_{4 k}, u_{4 k+1}, u_{4 k+2}, u_{4 k+3}, u_{4 k+4}\right\}
$$

such that $W$ contains both the two neighbors of $y$ in $Q_{1} \cup Q_{5}$. Then $\left|W^{*}\right| \geqslant 8+t$ and vertices in $W^{*}$ are adjacent to at most $t+2$ components of $G-W$. Since $t \leqslant 8$, $t+2<\frac{2}{3}(8+t)+1$. This gives a contradiction to Claim 5.2.

Claim 5.4. Let $x \in V(\mathcal{H})$ such that $x$ is a neighbor of some vertex in $\left\{u_{1}, u_{2}, \cdots, u_{4 k+4}\right\}$. Then $x \notin W$.

Proof. Assume to the contrary and without loss of generality, that $x \in V\left(Q_{1}\right) \cap W$ is a neighbor of $u_{1}$. (The argument for all other cases follows a similar idea, and if $x$ is a neighbor of a vertex in $\left\{u_{4 k-3}, u_{4 k-2}, \cdots, u_{4 k+4}\right\}$, we replace $k-1$ in the following argument by 8.) Since the neighbors of $x$ in $Q_{1}$ form a clique in $G, x$ is adjacent to exactly two components of $G-W$ by Claim 5.2, where one of the two components contains some neighbors of $x$ in $Q_{1}$, and the other of the two contains the vertex $u_{1}$. Let the neighbor of $u_{i}$ in $Q_{1}$ be $a_{i}$, and the neighbor of $u_{i}$ in $Q_{2}$ be $b_{i}$, for $i=1,2, \cdots, k-1$. By this labeling of the vertices, $x=a_{1}$. Let $W^{*}=W \cap$ $\left\{a_{1}, a_{2}, \cdots, a_{k-1}, b_{1}, b_{2}, \cdots, b_{k-1}\right\}$. To separate $u_{1}$ from some neighbors of $x$ contained in $Q_{1}$, since $W \cap T=\emptyset$ by Claim 5.3, we have that $\left|W^{*}\right| \geqslant 2$. We assume, without loss of generality, that $a_{1}, a_{2}, \cdots, a_{p} \in W, b_{1}, b_{2}, \cdots, b_{p} \notin W, a_{p+1}, a_{p+2}, \cdots, a_{p+q} \notin W$, $b_{p+1}, b_{p+2}, \cdots, b_{p+q} \in W$, and $a_{p+q+1}, b_{p+q+1}, \cdots, a_{p+q+r}, b_{p+q+r} \in W$ for some integers $p, q, r$ with $1 \leqslant p, q, r \leqslant k-1$ and $p+q+r \leqslant k-1$. Then $\left|W^{*}\right|=p+q+2 r$, and vertices in $W^{*}$ are adjacent to in total at most $r+2$ components in $G-W$, and they are adjacent to in total exactly $r+2$ components in $G-W$ only if $p+q+r=k-1$. If $r \geqslant 3$, then $r+2<\frac{2}{3}(2 r)+1 \leqslant \frac{2}{3}(p+q+2 r)+1$. This gives a contradiction to Claim 5.2. So we assume that $r \leqslant 2$. If $p+q+r \geqslant 3$, then since $r \leqslant 2$, we get $r+2<\frac{2}{3}(p+q+2 r)+1$, showing a contradiction to Claim 5.2 again. Thus, we have that $p+q+r \leqslant 2<k-1$, as $k \geqslant 7$. This particularly implies that vertices in $W^{*}$ are adjacent to at most $r+1$ components in $G-W$. As $\left|W^{*}\right| \geqslant 2$, Claim 5.2, together with the argument above that vertices in $W^{*}$ are adjacent to at most $r+1$ components in $G-W$, implies that $r=2$. However, $r+1=3<\frac{8}{3}+1 \leqslant \frac{2}{3}(p+q+2 r)+1$. Again, we achieve a contradiction to Claim 5.2.

Let the neighbors of vertices $u_{4 k+5}, u_{4 k+6}, \cdots, u_{6 k+1}$ in $\mathcal{H}$ be $x_{1}, x_{2}, \cdots, x_{2 k-3}$, respectively. Now by Claim 5.3 and Claim 5.4, we have that $W \subseteq S \cup\left\{x_{1}, x_{2}, \cdots, x_{2 k-3}\right\}$. 
Assume that $\left|W \cap\left\{x_{1}, x_{2}, \cdots, x_{2 k-3}\right\}\right|=t$. Then $G-W$ has exactly $t+1$ components, and since $t \leqslant 2 k-3$, we get

$$
\frac{|W|}{c(G-W)}=\frac{k+t}{t+1} \geqslant \frac{3}{2}
$$

This gives a contradiction to the assumption that $\tau(G)<\frac{3}{2}$.

\section{Acknowledgements}

The authors are grateful to the anonymous referee for the helpful comments. In particular, for suggesting us to provide the example given in Section 5.

\section{References}

[1] R. E. L. Aldred, Yoshimi Egawa, Jun Fujisawa, Katsuhiro Ota, and Akira Saito. The existence of a 2 -factor in $K_{1, n}$-free graphs with large connectivity and large edge-connectivity. J. Graph Theory, 68(1):77-89, 2011.

[2] Gunnar Brinkmann, Sheshayya A. Choudum, Stefan Grünewald, and Eckhard Steffen. Bounds for the independence number of critical graphs. Bull. London Math. Soc., 32(2):137-140, 2000.

[3] Yan Cao, Guantao Chen, Suyun Jiang, Huiqing Liu, and Fuliang Lu. Hamiltonicity of edge-chromatic critical graphs. arXiv:1708.08921, 2017.

[4] Guantao Chen, Akira Saito, and Songling Shan. The existence of a 2-factor in a graph satisfying the local Chvátal-Erdös condition. SIAM J. Discrete Math., 27(4):17881799, 2013.

[5] Guantao Chen and Songling Shan. Vizing's 2-factor conjecture involving large maximum degree. Journal of Graph Theory, 86(4): 422-438, 2017.

[6] Hikoe Enomoto, Bill Jackson, P. Katerinis, and Akira Saito. Toughness and the existence of $k$-factors. J. Graph Theory, 9(1):87-95, 1985.

[7] Stefan Grünewald and Eckhard Steffen. Independent sets and 2-factors in edgechromatic-critical graphs. J. Graph Theory, 45(2):113-118, 2004.

[8] Ian Holyer. The NP-completeness of edge-coloring. SIAM J. Comput., 10(4):718-720, 1981.

[9] Rong Luo, Zhengke Miao, and Yue Zhao. Hamiltonian cycles in critical graphs with large maximum degree. Graphs Combin., 32(5):2019-2028, 2016.

[10] Rong Luo and Yue Zhao. A note on Vizing's independence number conjecture of edge chromatic critical graphs. Discrete Math., 306(15):1788-1790, 2006.

[11] Rong Luo and Yue Zhao. A new upper bound for the independence number of edge chromatic critical graphs. J. Graph Theory, 68(3):202-212, 2011. 
[12] Rong Luo and Yue Zhao. A sufficient condition for edge chromatic critical graphs to be Hamiltonian - an approach to Vizing's 2-factor conjecture. J. Graph Theory, 73(4):469-482, 2013.

[13] W. T. Tutte. A short proof of the factor theorem for finite graphs. Canadian J. Math., 6:347-352, 1954.

[14] V. G. Vizing. The chromatic class of a multigraph. Kibernetika (Kiev), 1965(3):2939, 1965.

[15] V. G. Vizing. Critical graphs with given chromatic class. Diskret. Analiz No., 5:9-17, 1965.

[16] V. G. Vizing. Some unsolved problems in graph theory. Uspehi Mat. Nauk, 23(6 (144)):117-134, 1968.

[17] Douglas R. Woodall. The independence number of an edge-chromatic critical graph. J. Graph Theory, 66(2):98-103, 2011. 